\title{
Scaling of maximum strength with grain size in nanotwinned fcc metals
}

\author{
Yujie Wei* \\ State Key Laboratory of Nonlinear Mechanics, Institute of Mechanics, Chinese Academy of Sciences, Beijing 100190, P.R. China
}

(Received 3 January 2011; revised manuscript received 27 February 2011; published 21 April 2011)

\begin{abstract}
Engineers usually face the difficulty of making careful choices between one good and another in terms of strength, ductility, and electrical conductance in nanostructured metals. The emergence of polycrystalline $\mathrm{Cu}$ with ultrafine grains and the included nanotwins supplies an ideal solution because such materials owe high strength, high electrical conductance, and intermediate ductility. We answer in this Brief Report where the strength of such materials maximizes and how it depends on grain size. Based on the competitive plastic deformation mechanisms - the strengthening mechanism by inclined dislocations (with respect to twin planes) and detwinning-induced softening-we find that the critical twin thickness where the strength maximizes is proportional to $d^{1 / 2}$ and the maximum strength is proportional to $d^{-1 / 2}$.
\end{abstract}

DOI: 10.1103/PhysRevB.83.132104

PACS number(s): 62.20.F-, 62.25.-g, 62.23.St

Commonly seen polycrystalline metals are aggregates of small grains and the resultant grain boundaries (GBs) play a central role in determining the physical properties of such materials. Mechanically, GBs block the motion of dislocations, and the consequence of this is described by the well-known Hall-Petch strengthening. ${ }^{1,2}$ Along with the gain in strength in polycrystals as their grain sizes reduce, their other properties like deformability and thermal and electric conductivities are known to decrease..$^{3-5}$ The situation becomes worse in nanostructured materials because of the presence of a significant volume fraction of disordered GBs. Now the question arises: Do we have to make careful choices between one good and another in terms of strength, ductility, thermal conductivity, and electrical conductivity in nanostructured metals? Recent success in synthesizing dense nanotwins in ultrafine grains, ${ }^{4,6-11}$ let us expect more. In comparison to conventional coarse-grained $\mathrm{Cu}$, such nanotwinned $\mathrm{Cu}$ has high strength, intermediate ductility, and almost no change in electrical conductivity. ${ }^{4} \mathrm{Lu}$ et al. further revealed ${ }^{12}$ that there exists a maximum strength in nanotwinned samples with fixed grain size $d \approx 500 \mathrm{~nm}$ when twin thicknesses $\lambda$ vary from $90 \mathrm{~nm}$ down to $4 \mathrm{~nm}$. The maximum strength occurs in the sample with $\lambda \approx 15 \mathrm{~nm}$. The deformation mechanisms for the subsequent strength softening in nanotwinned $\mathrm{Cu}$ as $\lambda$ decreases from 15 to $4 \mathrm{~nm}$ are different from what happens in general nanocrystalline metals as their grain sizes reduce from about $10 \mathrm{~nm}$. For the latter, excessive GB volume as grain size reduces can facilitate GB diffusion ${ }^{13}$ and GB sliding, ${ }^{3,14-16}$ which weakens materials when their grain sizes reduce to some point. ${ }^{17-21}$ In nanotwinned $\mathrm{Cu}$, however, softening by GB-associated deformation can be ruled out since the average grain sizes of the samples are almost the same while their twin thicknesses change. Large-scale molecular dynamics (MD) simulations ${ }^{22}$ and theoretical analysis, ${ }^{22,23}$ show that the strengthening to softening transition as twin thickness reduces originates from the competition between dislocations inclined to twin boundaries (TBs) with those parallel to TBs. Partial dislocations in samples with narrow twins are predominantly residing in twin planes and moving parallel to twin planes (Fig. 1(a)), and we will refer to them as twinning partials hereafter. Inclined partial dislocations are observed to be the primary plastic carrier in samples with wide twins (Fig. 1(b)).
Twinning partials mediated detwinning, which accounts for the strength softening. ${ }^{22}$ The work in Refs. 22 and 23 also showed that the critical twin thickness $\lambda_{T}$ where the strengths of the nanotwinned metals maximized depends on grain size. We show in this work that the transitional twin thickness follows $\lambda_{T} \propto d^{1 / 2}$, and the corresponding maximum strength is proportional to $d^{-1 / 2}$.

Since GB deformation can be excluded for the strength softening in nanotwinned $\mathrm{Cu}$, the critical twin thickness is believed to be in the regime when the primary plastic deformation carriers change from the inclined dislocations to detwinning as twin thickness reduces. We start to formulate the respective critical shear stresses to activate the two types of dislocation activities. The detwinning process mediated by twinning partials is regarded as a shear transformation in an ellipsoid with a characteristic shear strain $e^{T}=0.707$ in fcc metals. We use Eshelby's shear transformation idea ${ }^{24}$ to approximate the increment in strain energy by detwinning, which differs from the microscopic methodology of computing the increasing strain energy by summing the self-energies from all discrete twinning partials as well as their interaction energies in the detwinned region. The singular stress/strain fields induced by twinning partials are homogenized and the collective strain field from dislocations is replaced by a uniform shear transformation in the detwinned region. This treatment circumvents the difficulty to calculate the interaction energy between twinning partials within the distance comparable to their Burger's vector in the microscopic discrete dislocation approach. A similar idea has been applied to martensitic phase transformation ${ }^{25-27}$ and precipitate twinning. ${ }^{28}$ Based on the energy criterion, the required critical resolved shear stress to detwin is related to twin thicknesses and grain sizes as ${ }^{23}$

$$
\tau-\tau_{l}=\alpha \frac{\pi}{8} \frac{2-v}{1-v} \frac{\lambda}{d} \mu e^{T}+\psi \frac{\gamma_{\mathrm{tb}}}{\lambda e^{T}},
$$

where $\tau_{l}$ is the lattice resistance to the motion of twinning partials in a twin plane, $\alpha$ is a coefficient on the order of unit, $\nu$ and $\mu$ are the Poisson's ratio and the shear modulus, respectively, $\gamma_{\mathrm{tb}}$ is the energy of a twin boundary, and $\psi$ is a coefficient, $\psi=-2$ for the complete detwinning of a TB, $\psi=2$ for the formation of a TB, and $\psi=0$ for the thickening or thinning of a TB. In the circumstance that plasticity is 


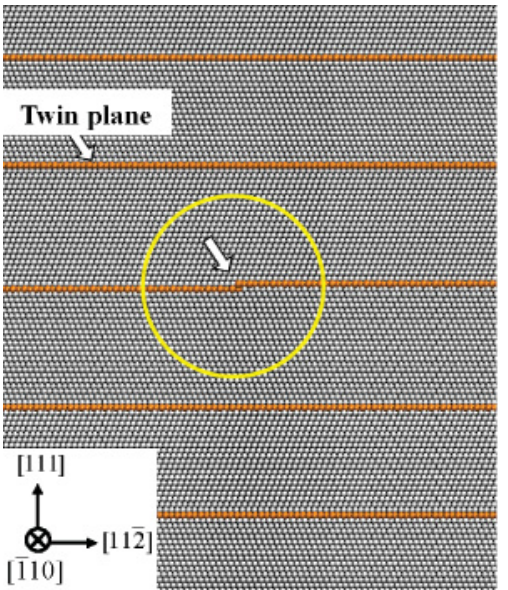

(a)

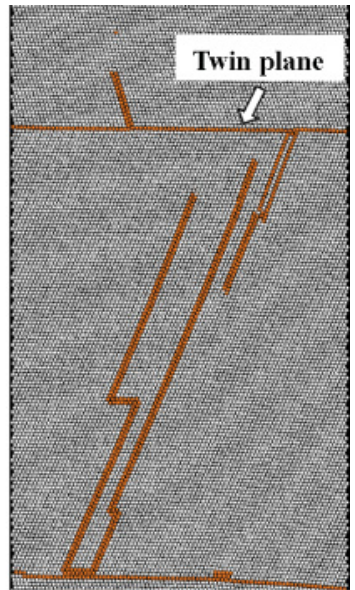

(b)
FIG. 1. (Color online) Planar view of typical nucleated partial dislocations in nanotwinned fcc metals from molecular dynamics simulations (gray: atoms in fcc lattice; orange: atoms of other type). Snapshots are abstracted from MD simulations on polycrystalline nanotwinned $\mathrm{Cu}$ reported in Ref. 22, where details about those simulations could be found. (a) A twinning partial (indicated by the arrow in the cycle) residing in twin planes and moving parallel to the twin planes in a sample with narrow twins; (b) inclined partial dislocations (with respect to twin planes) in a sample with wide twins.

exclusively accommodated for by detwinning, Eq. (1) predicts that the strength in nanotwinned metals softens as the twin thickness $\lambda$ decreases, which qualitatively agrees with the tensile experiments. ${ }^{12}$ The puzzling inverse size effect that a higher population of deformation twins occur in bigger grains in nanocrystalline materials ${ }^{29}$ can be readily explained using Eq. (1). ${ }^{23}$ We note that the kinetics model ${ }^{22}$ and energetic model $^{23}$ for detwinning in nanotwinned $\mathrm{Cu}$ are not necessarily two different viewpoints, but rather refer to the nucleation and growth aspects of the whole deformation process. It is desirable to consider these two aspects for detwinning in nanotwinned $\mathrm{Cu}$ since nucleation of twinning partials and the resultant detwinning by their propagation are naturally coupled processes. By using the energetic model alone, we essentially neglect the possible strain rate effects in nanotwinned $\mathrm{Cu}$.

Dislocations inclined to TBs are usually generated by emissions from GBs, via the cross slip, or through the interactions between dislocations with twin planes. ${ }^{30}$ It is found that the type of emitted dislocations, in general, depends on two length scales $\lambda_{c}$ and $l$, where the former is determined by material constants and the latter is a microstructure-dependent distance. Typically, $l$ is the characteristic distance between the dislocation nucleation sites to their front barrier (e.g., other dislocation walls, precipitates, and grain boundaries). When $l<\lambda_{c}$, nucleated dislocations are primarily of a partial type and complete dislocations dominate if $l>\lambda_{c}$. For nanocrystalline metals, $l$ approximates to the average grain size; in nanotwinned metals $l \approx \lambda$. When $\lambda<\lambda_{c}$, the critical resolved shear stress $\tau_{s}$ needed to trigger the nucleation of partial dislocations is ${ }^{31-34}$ given as

$$
\tau_{s}=\frac{1}{n}\left(\frac{\gamma_{\mathrm{sf}}}{b}+\frac{\mu b^{(c)}}{3 \lambda}\right),
$$

where $n$ is a geometrical parameter, $b$ and $b^{(c)}$ are the magnitudes of the Burger vectors of partial and complete dislocations, respectively, and $\gamma_{\mathrm{sf}}$ is the stacking fault energy. If $\lambda>\lambda_{c}$, corresponding $\tau_{s}$ to nucleate complete dislocations is given as

$$
\tau_{s}=\frac{\mu b^{(c)}}{n \lambda}
$$

It is easy to see that $\lambda_{c}$ can be obtained by comparing Eq. (2a) to Eq. (2b). Based on the estimate given by Asaro and Suresh, ${ }^{33} \lambda_{c}$ is about $40 \mathrm{~nm}$ for $\mathrm{Cu}$. We hence use Eq. (2a) to fit the strengthening regime of $\mathrm{nt}-\mathrm{Cu}$ as $\lambda$ changes from about 50 to $15 \mathrm{~nm}$. The applications of Eqs. (1) and (2a) to the existing experimental data ${ }^{12}$ give $\alpha=0.4$ and $n=1.8$, respectively, as seen in Fig. 2. We note that the same $\alpha$ and $n$ obtained here will be applied to discussions hereafter.

Equations (1) and (2a) are derived under the assumptions that either twinning partials or dislocations inclined to TBs are the exclusive plastic deformation mechanism, which may not be true for compatible deformation in individual grains. Based on Taylor's least work hypothesis, ${ }^{35}$ Chin et al. ${ }^{36}$ suggested that during the accommodation of constrained deformation in fcc metals by slip and twinning, the operation of slip and twinning systems is found by minimizing the orientation factor

$$
M=\frac{1}{\varepsilon_{x x}}\left(\sum_{i} s_{i}+\beta \sum_{j} t_{j}\right),
$$

where $s_{i}$ and $t_{j}$ are the amounts of shear in the $i$ th operative slip system and the $j$ th operative twinning system, respectively, $\beta=\tau / \tau_{s}$ is the ratio of the critical resolved shear stress for twinning versus slip, and $\epsilon_{x x}$ is the imposed uniaxial strain. Chin et al. ${ }^{36}$ obtained that when

$$
\frac{\sqrt{3}}{3}<\beta<\frac{2 \sqrt{3}}{3},
$$



FIG. 2. (Color online) Yield strength (experiments and modeling) versus twin thickness for nt-Cu. Experimental data replotted from Ref. 12, theoretical data from Eqs. (1) and (2b), where one parameter has been fitted ( $\alpha$ and $n$, respectively). The critical shear strengths are multiplied by the Taylor factor of 3 to convert them to tensile strengths. 
both twinning and the inclined dislocation slip are active. In $\mathrm{nt}-\mathrm{Cu}$, we also suggest that the transition from inclined dislocations to detwinning happens when the ratio of $\beta$ falls in the region given by Eq. (3) in nanotwinned $\mathrm{Cu}$. Therefore $\beta=\tau / \tau_{s}$ can be obtained by substituting $\tau$ and $\tau_{s}$ in Eq. (3) by Eqs. (1) and (2a), respectively. The maximum strength occurs when both twinning and the inclined dislocation slip are active. We hence yield the transitional twin thickness $\lambda_{T}$ as

$$
n\left(\alpha \frac{\pi}{8} \frac{2-v}{1-v} \frac{\lambda_{T}}{d} \mu e^{T}\right) /\left(\frac{\gamma_{\mathrm{sf}}}{b}+\frac{\mu b^{(c)}}{3 \lambda_{T}}\right) \approx 1 .
$$

Note that we have neglected the lattice resistance $\tau_{l}$ and the TB energy term since both of them are on the order of $1 \mathrm{MPa}$ and have the opposite sign. For nt-Cu with $d<1000 \mathrm{~nm}$, we have

$$
\frac{\gamma_{\mathrm{sf}}^{2}}{b^{2}} \ll \frac{\alpha n \pi}{6} \frac{2-v}{1-v} \frac{\mu^{2} b^{(c)}}{d} e^{T},
$$

and Eq. (4) can be rewritten as

$$
\lambda_{T}=a_{0} \frac{\gamma_{\mathrm{sf}} d}{\mu b}+a_{1} \sqrt{b^{(c)} d},
$$

with

$$
a_{0}=\frac{4}{\alpha n \pi e^{T}} \frac{1-v}{2-v}, \quad a_{1}=\sqrt{\frac{8}{3 \alpha n \pi e^{T}} \frac{1-v}{2-v}} .
$$

Using the corresponding material parameters given in Table I for $\mathrm{Cu}$, we obtain $a_{0}=1$ and $a_{1}=0.8$. Since $a_{0} \gamma_{\mathrm{sf}} / \mu b$ is about $3.7 \times 10^{-4}$ and the first term in Eq. (5) is less than $0.5 \mathrm{~nm}$ if $d<1000 \mathrm{~nm}$, we may neglect it without loss of accuracy. The transitional twin thickness $\lambda_{T}$ is now simplified to be

$$
\lambda_{T}=a_{1} \sqrt{b^{(c)} d}
$$

We have plotted Eq. (6) against the experimental results ${ }^{12}$ and the MD simulations ${ }^{22}$ in Fig. 3. An excellent match between the model prediction and the data from both experiments and MD simulations is seen.

TABLE I. Material constants for nanotwinned $\mathrm{Cu}$ and the value of fitting parameters $\alpha$ and $\mathrm{n}$ in Eqs. (1) and (2a), respectively. Theoretical curves in Figs. 2 to 4 are all produced by using the same values listed here.

\begin{tabular}{lcc}
\hline \hline Description of parameter & Symbol & Value \\
\hline Shear modulus of Cu at room temperature & $\mu$ & $45 \mathrm{GPa}$ \\
Poisson's ratio & $v$ & 0.35 \\
Grain size & $d$ & $500 \mathrm{~nm}$ \\
Transformation shear strain & $e^{T}$ & 0.707 \\
Twin boundary energy & $\gamma_{\mathrm{tb}}$ & $24 \mathrm{~mJ} / \mathrm{m}^{2}$ \\
Stacking fault energy & $\gamma_{\mathrm{sf}}$ & $45 \mathrm{~mJ} / \mathrm{m}^{2}$ \\
Index parameter & $\psi$ & -2 \\
Transitional grain size & $\lambda_{c}$ & $\sim 40 \mathrm{~nm}$ \\
Burgers vector of a partial dislocation & $b$ & $1.49 \mathrm{~nm}$ \\
Burgers vector of a complete dislocation & $b^{(c)}$ & $2.58 \mathrm{~nm}$ \\
Geometrical parameter & $n$ & 1.8 \\
Geometrical factor & $\alpha$ & 0.4 \\
\hline \hline
\end{tabular}

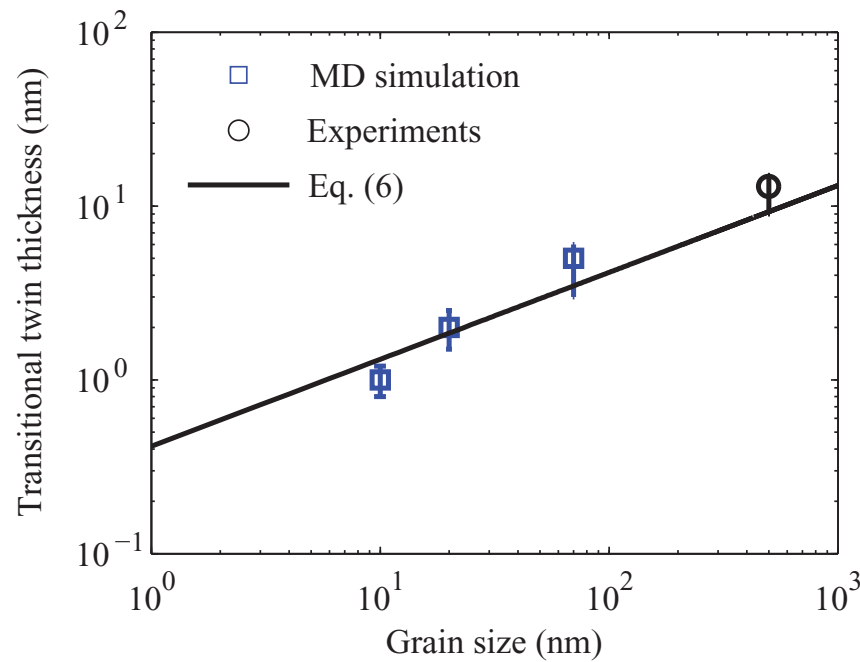

FIG. 3. (Color online) Predicted grain size versus the transitional twin thickness where the strength nt-Cu maximizes. The prediction by Eq. (6) that matches well with the experimental data comes from Ref. 12 and those from MD simulations Ref. 22.

Inserting Eq. (6) into Eq. (2a), we obtain the achievable maximum strength $\tau_{\max }$ in nanotwinned fcc metals with grain size $d$,

$$
\tau_{\max }=\frac{1}{n} \frac{\gamma_{\mathrm{sf}}}{b}+\frac{\mu}{3 n a_{1}}\left(\frac{b^{(c)}}{d}\right)^{1 / 2} .
$$

A similar relationship can be obtained if one substitutes Eq. (6) into Eq. (1). It is noted that Eq. (7) resembles the classic Hall-Petch strengthening relation although the corresponding deformation mechanisms which give rise to such a relationship are distinct. Figure 4 gives the grain size versus the maximum strength in nanotwined fcc metals described by Eq. (7). The prediction from the theory captures the experiments ${ }^{12}$ and the MD simulations ${ }^{22}$ very well.

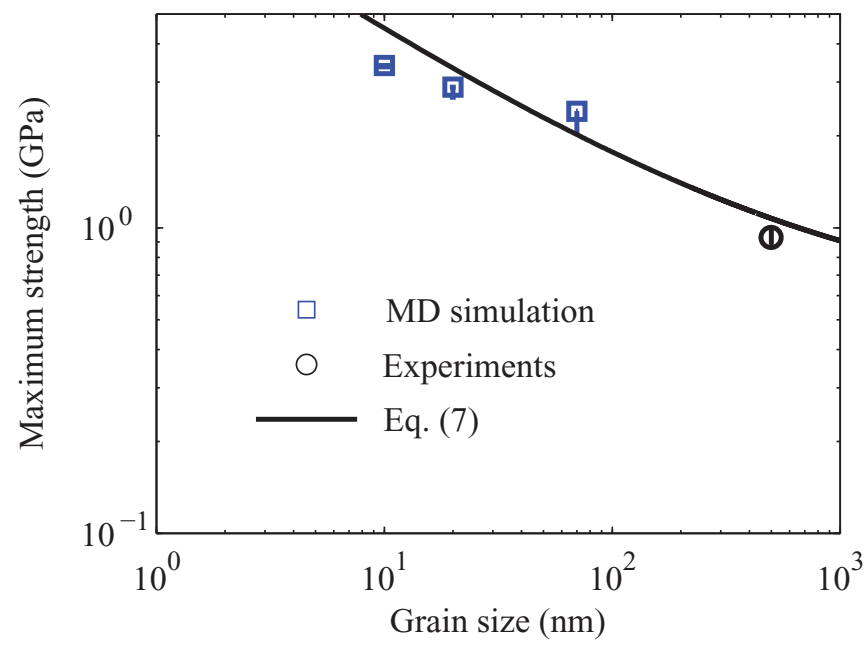

FIG. 4. (Color online) Predicted grain size versus the maximum strength in nt-Cu using Eq. (7) that matches well with the experimental data comes from Ref. 12 and those from MD simulations Ref. 22. 
In summary, we show that the critical twin thickness $\lambda_{T}$ where the strength of nanotwinned fcc metals maximizes at a given grain size $d$ is proportional to $d^{1 / 2}$. Such a scaling law shows excellent agreement with available experiments and large-scale molecular dynamics simulations where the transitional twin thicknesses were reported. In return, the maximum strength $\tau_{\max }$ in nanotwinned samples at a given grain size $d$ is found to follow $\tau_{\max } \propto d^{-1 / 2}$. The predicted transitional twin thickness where the strength of such material maximizes can serve as a meaningful guidance for material synthesis.

\section{ACKNOWLEDGMENTS}

Y.W. is grateful to the Chinese Academy of Sciences for financial support via the "Hundred Talent program" and support from NSFC via Grant No. 11021262. *yujie_wei@lnm.imech.ac.cn

${ }^{1}$ E. O. Hall, Proc. Phys. Soc. London, Sect. B 56, 747 (1951).

${ }^{2}$ N. J. Petch, J. Iron Steel Inst. 174, 25 (1953).

${ }^{3}$ H. Gleiter, Prog. Mater. Sci. 33, 223 (1989).

${ }^{4}$ L. Lu, Y. Shen, X. Chen, L. Qian, and K. Lu, Science 304, 422 (2004).

${ }^{5}$ K. Lu, L. Lu, and S. Suresh, Science 324, 349 (2009).

${ }^{6}$ M. D. Merz and S. D. Dahlgren, J. Appl. Phys. 46, 3235 (1975).

${ }^{7}$ X. Zhang, A. Misra, H. Wang, T. E. Mitchell, M. Nastasi, J. P. Hirth, J. D. Embury, and R. G. Hoagland, Acta Mater. 52, 995 (2004).

${ }^{8}$ X. Zhang, A. Misra, H. Wang, X. H. Chen, L. Lu, K. Lu, and R. G. Hoagland, Appl. Phys. Lett. 88, 173116 (2006).

${ }^{9}$ A. M. Hodge, Y. M. Wang, and T. W. Barbee Jr., Mater. Sci. Eng. A 429, 272 (2006).

${ }^{10}$ A. M. Hodge, Y. M. Wang, and T. W. Barbee Jr., Scr. Mater. 59, 163 (2008).

${ }^{11}$ C. J. Shute, B. D. Myers, S. Xie, T. W. Barbee Jr., A. M. Hodge, and J. R. Weertman, Scr. Mater. 60, 1073 (2009).

${ }^{12}$ L. Lu, X. Chen, X. Huang, and K. Lu, Science 323, 607 (2009).

${ }^{13}$ R. I. Coble, J. Appl. Phys. 34, 1679 (1963).

${ }^{14}$ D. Wolf, V. Yamakov, S. R. Phillpot, A. K. Mukherjee, and H. Gleiter, Acta Mater 53, 1 (2005).

${ }^{15}$ M. A. Meyers, A. Mishra, and D. J. Benson, Prog. Mater. Sci. 51, 427 (2006).

${ }^{16}$ Y. J. Wei, A. F. Bower, and H. J. Gao, Acta Mater. 56, 1741 (2008).

${ }^{17}$ A. H. Chokshi, A. Rosen, J. Karch, and H. Gleiter, Scr. Metall. Mater. 23, 1679 (1989).
${ }^{18}$ K. Lu and M. L. Sui, Scr. Metall. Mater. 28, 1465 (1993).

${ }^{19}$ S. Yip, Nature (London) 391, 532 (1998).

${ }^{20}$ J. Schiøtz, F. D. Di Tolla, and K. W. Jacobsen, Nature (London) 391, 561 (1998).

${ }^{21}$ H. Van Swygenhoven, M. Spaczer, A. Caro, and D. Farkas, Phys. Rev. B 60, 22 (1999).

${ }^{22}$ X. Y. Li, Y. J. Wei, L. Lu, K. Lu, and H. J. Gao, Nature (London) 464, 877 (2010).

${ }^{23}$ Y. J. Wei, Mater. Sci. Eng. A 528, 1558 (2011).

${ }^{24}$ J. D. Eshelby, Proc. R. Soc. A 243, 376 (1957).

${ }^{25}$ J. W. Christian, Acta Metall. 6, 377 (1958).

${ }^{26}$ J. W. Christian and S. Mahajan, Prog. Mater. Sci. 39, 1 (1995).

${ }^{27}$ T. Waitz, T. Antretter, F. D. Fischer, N. K. Simha, and H. P. Karnthaler, J. Mech. Phys. Solids 55, 419 (2007).

${ }^{28}$ F. Appel, F. D. Fischer, and H. Clemens, Acta Mater. 55, 4915 (2007).

${ }^{29}$ X. L. Wu and Y. T. Zhu, Phys. Rev. Lett. 101, 025503 (2008).

${ }^{30}$ J. P. Hirth and J. Lothe, Theory of Dislocations, 2nd ed. (John Wiley \& Sons, New York, 1982).

${ }^{31}$ R. J. Asaro, P. Krysl, and B. Kad, Philos. Mag. Lett. 83, 733 (2003).

${ }^{32}$ B. Zhu, R. J. Asaro, P. Krysl, and R. Bailey, Acta Mater. 53, 4825 (2005).

${ }^{33}$ R. J. Asaro and S. Suresh, Acta Mater. 53, 3369 (2005).

${ }^{34}$ Y. J. Wei, C. Su, and L. Anand, Acta Mater. 54, 3177 (2006).

${ }^{35}$ G. I. Taylor, J. Inst. Met. 62, 307 (1938).

${ }^{36}$ G. Y. Chin, W. F. Hosford, and D. R. Mendorf, Proc. R. Soc. A 309, 433 (1969). 